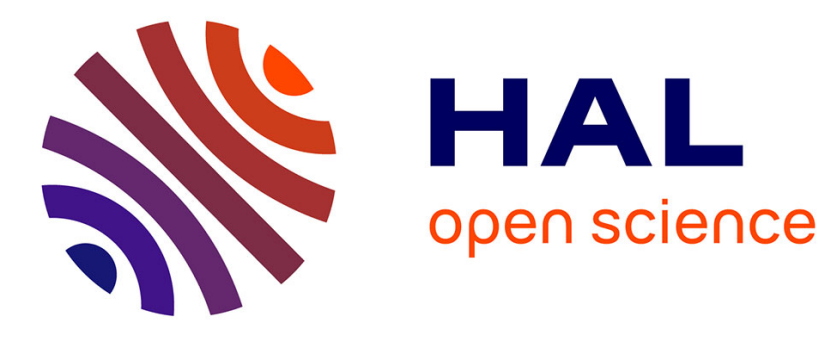

\title{
Improving the Usability of a MAS DSML
}

\author{
Tomaz Miranda, Moharram Challenger, Baris Tekin Tezel, Omer Alaca, \\ Ankica Barisic, Vasco Amaral, Miguel Goulão, Geylani Kardas
}

\section{To cite this version:}

Tomaz Miranda, Moharram Challenger, Baris Tekin Tezel, Omer Alaca, Ankica Barisic, et al.. Improving the Usability of a MAS DSML. Danny Weyns; Viviana Mascardi; Alessandro Ricci. Engineering Multi-Agent Systems. 6th International Workshop, EMAS 2018, Stockholm, Sweden, July 14-15, 2018, Revised Selected Papers, 11375, Springer, pp.55-75, 2019, Lecture Notes in Computer Science, 978-3-030-25692-0. 10.1007/978-3-030-25693-7_4 . hal-03159960

\section{HAL Id: hal-03159960 https://hal.science/hal-03159960}

Submitted on 2 Jun 2021

HAL is a multi-disciplinary open access archive for the deposit and dissemination of scientific research documents, whether they are published or not. The documents may come from teaching and research institutions in France or abroad, or from public or private research centers.
L'archive ouverte pluridisciplinaire HAL, est destinée au dépôt et à la diffusion de documents scientifiques de niveau recherche, publiés ou non, émanant des établissements d'enseignement et de recherche français ou étrangers, des laboratoires publics ou privés. 


\title{
Improving the Usability of a MAS DSML
}

\author{
Tomás Miranda $^{1}$, Moharram Challenger ${ }^{2}$, Baris Tekin Tezel ${ }^{2,3}$, Omer Faruk \\ Alaca $^{2}$, Ankica Barišić ${ }^{1}$, Vasco Amaral ${ }^{1}$, Miguel Goulão ${ }^{1}$, and Geylani Kardas ${ }^{2}$ \\ 1 NOVA LINCS, DI, FCT, Universidade NOVA de Lisboa, Portugal \\ 2 International Computer Institute, Ege University, Izmir, Turkey \\ 3 Department of Computer Science, Dokuz Eylul University, Izmir, Turkey \\ tr.miranda@campus.fct.unl.pt, baris.tezel@deu.edu.tr, \\ omerfarukalaca@gmail.com, \{moharram.challenger, \\ geylani.kardas\}@ege.edu.tr, a.barisic@campus.fct.unl.pt, \{vma, \\ mgoul\}@fct.unl.pt
}

\begin{abstract}
Context: A significant effort has been devoted to the design and implementation of various domain-specific modeling languages (DSMLs) for the software agents domain.

Problem: Language usability is often tackled in an ad-hoc way, with the collection of anecdotal evidence supporting the process. However, usability plays an important role in the productivity, learnability and, ultimately, in the adoption of a MAS DSML by agent developers.

Method: In this chapter, we discuss how the principles of The "Physics" of Notations (PoN) can be applied to improve the visual notation of a MAS DSML, called SEA_ML and evaluate the result in terms of usability.

Results: The evolved version of the language, SEA_ML++, was perceived as significantly improved in terms of icons comprehensibility, adequacy and usability, as a direct result of employing the principles of PoN. However, users were not significantly more efficient and effective with SEA_ML++, suggesting these 2 properties were not chiefly constrained by the identified shortcomings of the SEA_ML concrete syntax.
\end{abstract}

Keywords: Usability $\cdot$ Multi-Agent Systems · Domain Specific Modeling Language $\cdot$ Physics of Notations $\cdot$ SEA_ML

\section{Introduction}

Software agents with the capability of both being autonomous and performing reactive/proactive behaviors, interact with each other in a Multi-agent system (MAS) to solve problems in a competitive or collaborative manner within an environment. To eliminate the complexity and the difficulty of MAS development, the researchers in agent-oriented software engineering (AOSE) field have significant efforts on design and implementation of various domain-specific modeling languages (DSMLs) such as DSML4MAS [19], FAML [4], SEA_ML [7], MASML [10, and JADEL [3. Those DSMLs are specific to the agent domain and 


\section{T. Miranda et al.}

provide appropriate integrated development environments (IDEs) in which both modelling and code generation for system-to-be-developed can be performed properly 25 .

To be effective, the proposed agent DSMLs need to meet the various stakeholder concerns and the related quality criteria for the corresponding MASs. Unfortunately, very often the evaluation of the DSML, especially covering the language components and the use of the DSML during design and implementation of agent-based systems, is completely missing or has been carried out with an idiosyncratic approach 8. Specifically, the usability, which plays an important role on the adoption of a MAS DSML by agent developers, needs to be taken into consideration preferably during language design and improved to better align the DSML with developer expectations. Hence, in this chapter, we focus on the usability of DSMLs for MAS and propose an approach for promoting the usability of such languages by applying the principles of The "Physics" of Notations $(\mathrm{PoN})$ 30. For this purpose, the visual notation of a MAS DSML, called SEA_ML 7], is evaluated and its usability is improved by employing each principle of PoN. Hence, it is possible to enrich SEA_ML's visual notation and its correlation to the linked semantic constructs. A comparative assessment of the improved language is also performed with 2 different experiments using end-users that are defined by the domain experts. SEA_ML is an open source language and it is easy to achieve both abstract and concrete syntax specifications. Moreover, reflecting the changes according to the conducted $\mathrm{PoN}$ experiments and generating the new version of the language become much easier since the required source code is available online. These are the main reasons of selecting SEA_ML as the application language in our work.

The rest of the chapter is organized as follows: Section 2 and Section 3 discuss SEA_ML and the principles of PoN respectively. The analysis of SEA_ML and improving its visual notation by using PoN principles are given in Section 4 . Comparative evaluation of the new language is discussed in Section 5. Related work is given in Section 6 and Section 7 concludes the chapter.

\section{SEA_ML}

SEA_ML [7] is a MAS modeling language which enables the developers to model agent systems in a platform independent level and then automatically generate codes and related documents required for the execution of the modeled MAS on target MAS implementation platforms. In addition to these capabilities, SEA_ML also supports the model-driven design and implementation of autonomous agents who can evaluate semantic data and collaborate with semantically-defined entities of the Semantic Web 35], like Semantic Web Services (SWSs). Within this context, it includes new viewpoints which specifically pave the way for the development of software agents working on the Semantic Web environment. Modeling agents, agent knowledge-bases, platform ontologies, SWS and interactions between agents and SWS are all possible in SEA_ML. 
To support MAS experts when programming their systems, and to be able to fine-tune them visually, SEA_ML covers all aspects of an agent system from the internal view of a single agent to the complex MAS organization.

To this end, SEA_ML's metamodel is divided into 8 viewpoints, each of which represents a different aspect for developing Semantic Web enabled MASs.

- Agent's Internal Viewpoint is related to the internal structures of semantic web agents (SWAs) and defines entities and their relations required for the construction of agents.

- Interaction Viewpoint expresses the interactions and the communications in a MAS by taking messages and message sequences into account.

- MAS Viewpoint solely deals with the construction of a MAS as a whole. It includes the main blocks which compose the complex system as an organization.

- Role Viewpoint delves into the complex controlling structure of the agents and addresses role types.

- Environmental Viewpoint describes the use of resources and interaction between agents with their surroundings.

- Plan Viewpoint deals with an agent Plan's internal structure, which is composed of Tasks and atomic elements such as Actions.

- Ontology Viewpoint addresses the ontological concepts which constitute agent's knowledge-base (such as belief and fact).

- Agent-SWS Interaction Viewpoint defines the interaction of agents with SWS including the definition of entities and relations for service discovery, agreement and execution. A SWA executes the semantic service finder Plan (SS_FinderPlan) to discover the appropriate services with the help of a special type of agent called SSMatchMakerAgent who executes the service registration plan (SS_RegisterPlan) for registering the new SWS for the agents. After finding the necessary service, one SWA executes an agreement plan (SS_AgreementPlan) to negotiate with the service. After negotiation, a plan for service execution (SS_ExecutorPlan) is applied for invoking the service.

Appendix A lists the important SEA_ML concepts (meta-entities) and their brief descriptions for the comprehension of the corresponding visual notations used in the diagrams throughout this chapter.

SEA_ML instances are given as inputs to a series of modelto-model and model-to-text transformations to achieve executable artifacts of the system-tobe-built for JADEX 33 agent platform and semantic web service description documents conforming to Web Ontology Language for Services (OWL-S) ontology 28 .

To demonstrate the modeling and implementation environment provided by SEA_ML, let us consider the development of a MAS for stock exchange software in which Investor (Buyer and/or Seller), Broker and Stock Trade Manager agents take role in a computerized stock trading system. All of the user agents including investors and brokers cooperate with stock trade manager agent to access the stock market. Also, the user agents interact with each other, for instance, investor 
T. Miranda et al.

A and investor B can cooperate with a broker in order to exchange the stock for which the broker is an expert. Figure 1 is a screenshot taken from SEA_ML modeling environment which shows the modeling of such a stock exchange MAS which is composed of 6 semantic web agent instances, 1 trade manager, 2 brokers, and 3 investors. The given model only considers the overview of the system from SEA_ML MAS viewpoint. However, it is also possible to model all specifications and components of the system considering the other SEA_ML viewpoints again inside the same IDE. Interested readers may refer to 7 for an extensive discussion on SEA_ML and 24 for complete design and implementation of this agent-based stock exchange system with SEA_ML.

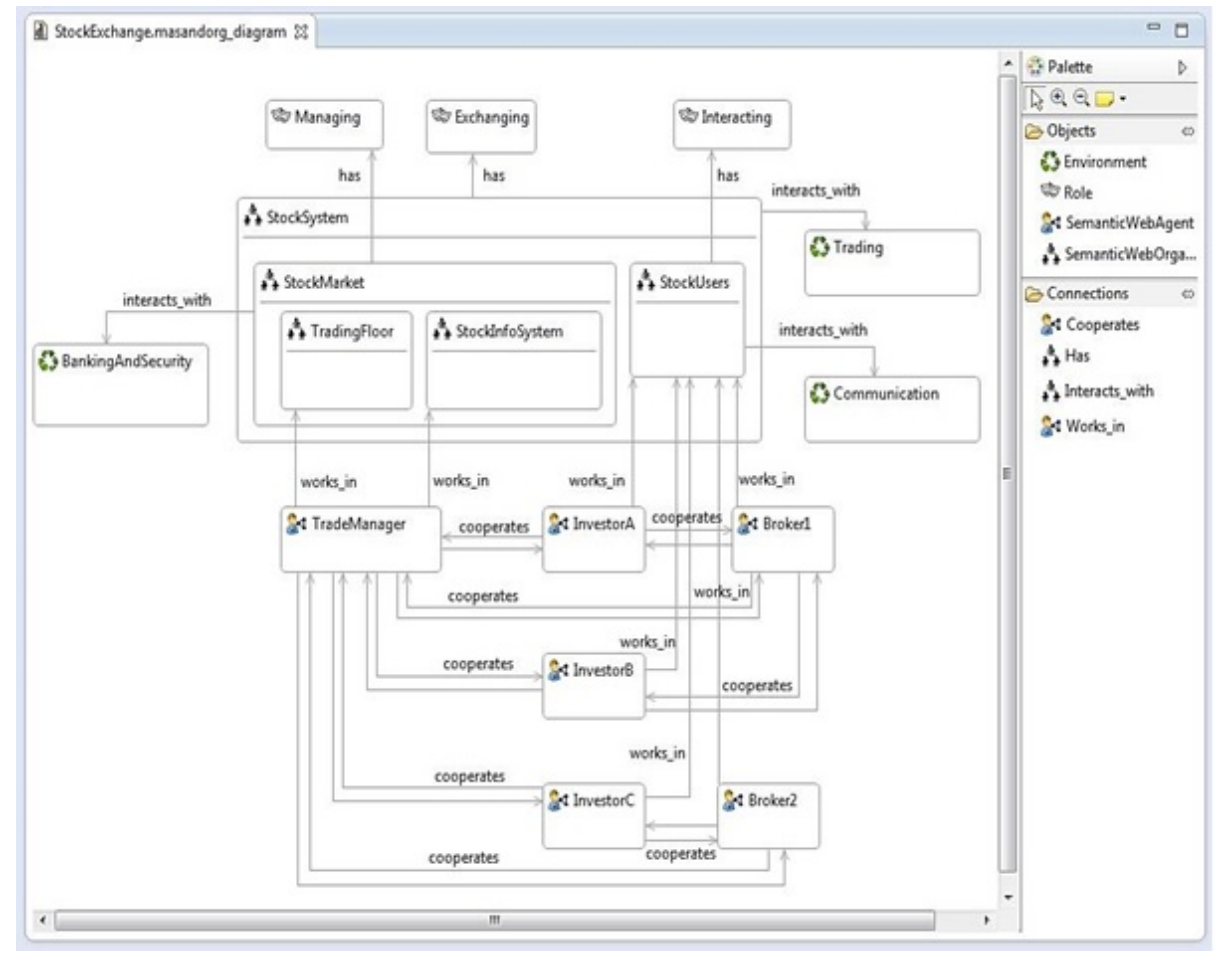

Fig. 1: MAS and Organization diagram for Stock Exchange System in SEA_ML

\section{Physics of Notations}

The Physics of Notations (PoN) 30 is a design theory which focuses on the perceptual (physical) properties of notations rather than their semantic (logical) properties. It is based on a set of 9 principles which form a prescriptive theory for designing cognitively effective visual notations, defined (and measured) as the 
Improving the Usability of a MAS DSML

Table 1: PoN principles

\begin{tabular}{|c|c|}
\hline Principle & Comment \\
\hline Semiotic Clarity & $\begin{array}{l}\text { There should be a } 1: 1 \text { correspondence between semantic } \\
\text { constructs and graphical symbols }\end{array}$ \\
\hline Perceptual Discriminability & $\begin{array}{l}\text { Different symbols should be clearly distinguishable from } \\
\text { each other }\end{array}$ \\
\hline Semantic transparency & $\begin{array}{l}\text { The appearance of visual representations should suggest } \\
\text { their meaning }\end{array}$ \\
\hline Complexity management & $\begin{array}{l}\text { Explicit mechanisms for dealing with complexity should } \\
\text { be included }\end{array}$ \\
\hline Cognitive integration & $\begin{array}{l}\text { There should be explicit mechanisms to support the } \\
\text { integration of information from different diagrams }\end{array}$ \\
\hline Visual expressiveness & $\begin{array}{l}\text { The full range of capacities and visual variables should } \\
\text { be used }\end{array}$ \\
\hline Dual coding & Text should be used to complement graphics \\
\hline Graphic economy & $\begin{array}{l}\text { The number of symbols presented in the notation may } \\
\text { affect the handling of the tool }\end{array}$ \\
\hline Cognitive fit & $\begin{array}{l}\text { Different dialects should be used for different tasks and } \\
\text { audiences. }\end{array}$ \\
\hline
\end{tabular}

speed, ease, and accuracy with which a given representation can be processed by the human mind. This principles can be used to evaluate, compare and ultimately enhance the communication properties of a given language when designing its visual concrete syntax.

The principles were synthesized from theory and empirical evidence about cognitive effectiveness of visual representations. Each principle was defined by its Name (named in a positive sense) and Semantic (theoretical) definition (A imperative statement of what it means), listed in Table 1 . Further, each principle contains Operational (empirical) definition, which gives evaluation procedures and/or metrics; Design strategies, Exemplars and Counter exemplars.

\section{Applying Physics of Notations Principles to SEA_ML}

We proposed notation improvements for all 8 viewpoints of SEA_ML by following 9 PoN principles given in Table 1. These improvements are employed in the development of the new version of SEA_ML, called SEA_ML++. Table 2 synthesizes the conclusions derived from review of $\mathrm{PoN}$ principles which is detailed in 29. Plus $(+)$ refers that SEA_ML currently conforms to the presented principle, while a minus (-) refers that SEA_ML has room for improvement under that principle.

SEA_ML notation conforms to Semiotic Clarity as for each SEA_ML viewpoint, different symbols are presented, representing a different semantic construct. There is no such case where two symbols represent the same semantic construct, or when they are not connected to a semantic construct. 
T. Miranda et al.

Table 2: Review of SEA_ML visual notation according to each principle of PoN

\begin{tabular}{|c|c|}
\hline Principle & Room for Improvement \\
\hline Semiotic Clarity & + \\
\hline Perceptual Discriminability & - \\
\hline Semantic Transparency & - \\
\hline Complexity Management & $+/-$ \\
\hline Cognitive Integration & + \\
\hline Visual Expressiveness & - \\
\hline Dual Coding & - \\
\hline Graphic Economy & + \\
\hline \multicolumn{2}{|c|}{ Cognitive Fit } \\
\hline OK - can be improved \\
\hline
\end{tabular}

Based on Perceptual Discriminability principle, SEA_ML can be improved, as some symbols only differ by a label, which is proven to be cognitively ineffective. The distance between visual symbols is too short, as predefined by the language editor when generating the tool.

Regarding Semantic Transparency principle we identify 19 visual notations of SEA_ML that could represent better intended meaning and provided improvement suggestions for each symbol (see [29]).

Complexity Management is not applicable, as SEA_ML does not have any direct mechanism for dealing with the complexity of the viewpoints.

SEA_ML conforms to Cognitive Integration principle, as it requires a name for every diagram and label used during the modelling. Every procedure is verified to be connected to some entity.

SEA_ML presents similar colours and symbols to similar semantic constructs. Some semantic figures are only differentiated by a letter, which is not conforming to Visual Expressiveness principle since the icons should be presented using different visual variables to automatically distinguish each semantic construct only looking to visual notation.

Based on the Dual Coding principle, SEA_ML has eleven visual notations that are only differentiated through letters or textual differences that are difficult to see, which are impossible to differentiate without it.

The user is presented with a palette of icons which are allowed to be used on each viewpoint, therefore conforming to the Graphic Economy principle.

Regarding Cognitive Fit, some of the proposed visual notation can be improved in order to have a better relation with other similar symbols presented on the SEA_ML language, which may turn the language easier to understand and to be worked for novice users.

Some SEA_ML visual notations were not modified as these notations reflect correctly its semantic constructs and they conform to $P_{o} N$ principles. Of the 43 symbols (44 including the symbol for arrows that relate each entity), 32 symbols were modified (see Figure 2 for the current (SEA_ML) and new (SEA_ML) notations). With respect to the proposed SEA_ML++ notation, the justification for each new symbol is defined below: 
Improving the Usability of a MAS DSML

\begin{tabular}{|c|c|c|}
\hline Concept & $\begin{array}{l}\text { Current } \\
\text { Notation }\end{array}$ & $\begin{array}{c}\text { New } \\
\text { Notation }\end{array}$ \\
\hline Goal & (๑) & \\
\hline Capability & & \\
\hline Fact & छ & \\
\hline Plan & 䨽 & $\sqrt[x y]{x=x}$ \\
\hline $\begin{array}{l}\text { Semantic Service } \\
\text { Register Plan }\end{array}$ & R & \\
\hline $\begin{array}{l}\text { Semantic Service } \\
\text { Finder Plan }\end{array}$ & & \\
\hline $\begin{array}{l}\text { Semantic Service } \\
\text { Agreement Plan }\end{array}$ & A & \\
\hline $\begin{array}{l}\text { Semantic Service } \\
\text { Executor Plan }\end{array}$ & $\mathbf{E}$ & \\
\hline Send & 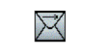 & \\
\hline Receive & $\sum$ & קה \\
\hline Action & & \\
\hline
\end{tabular}

\begin{tabular}{|c|c|c|}
\hline Concept & $\begin{array}{l}\text { Current } \\
\text { Notation }\end{array}$ & $\begin{array}{c}\text { New } \\
\text { Notation }\end{array}$ \\
\hline Message & 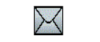 & \\
\hline $\begin{array}{l}\text { Message } \\
\text { Sequence }\end{array}$ & B & \\
\hline DomainRole & & 69 \\
\hline Agent State & & \\
\hline Resource & & \\
\hline Web Service & 0 & \\
\hline $\begin{array}{l}\text { Semantic Web } \\
\text { Service }\end{array}$ & & \\
\hline Grounding & & \\
\hline Process & & \\
\hline Interface & & \\
\hline Precondition & & \\
\hline
\end{tabular}

\begin{tabular}{|c|c|c|}
\hline Concept & $\begin{array}{l}\text { Current } \\
\text { Notation }\end{array}$ & $\begin{array}{c}\text { New } \\
\text { Notation }\end{array}$ \\
\hline Effect & (E) & \\
\hline $\begin{array}{l}\text { Architecture } \\
\text { Role }\end{array}$ & 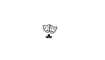 & \\
\hline $\begin{array}{l}\text { Ontology } \\
\text { Mediator Role }\end{array}$ & $\stackrel{-209}{\rightleftarrows}$ & \\
\hline $\begin{array}{l}\text { Semantic Web } \\
\text { Organization }\end{array}$ & 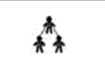 & \\
\hline Role Ontology & $\pi$ & \\
\hline $\begin{array}{l}\text { Organization } \\
\text { Ontology }\end{array}$ & $* *$ & \\
\hline $\begin{array}{l}\text { Service } \\
\text { Ontology }\end{array}$ & & (2) \\
\hline Interaction & & \\
\hline Behavior & $\rightarrow$ & \\
\hline Agent Type & 4 & \\
\hline & & \\
\hline
\end{tabular}

Fig. 2: SEA_ML vs SEA_ML++ notations

1. Goal - The new notation adds color to the target, making it more appropriate to be selected when using viewpoints that use this semantic construct;

2. Capability - The current visual notation may induce users wrong. The new notation reflects that users have a set of capabilities in order to solve their problems;

3. Fact - The current notation is similar to other notations present in SEA_ML. The new notation (check mark) reflects something that is correct and concrete;

4. Plan - The notation addresses a plan to reach a goal from $\mathrm{X}$ to $\mathrm{Y}$;

5. Semantic Service Register Plan (SSRP) - The current notation has 4 similar symbols, being distinguished through different letters. The new notation adds the SWS notation and a person registering to a customer's list;

6. Semantic Service Finder Plan (SSFP) - The current notation has 4 similar symbols, being distinguished through different letters. The new notation adds the "Semantic Web Services" notation and a magnifying glass;

7. Semantic Service Agreement Plan (SSAP) - The current notation has 4 similar symbols, being distinguished through different letters. The new notation adds the "Semantic Web Services" notation and a handshake between 2 people;

8. Semantic Service Executor Plan (SSEP) - The current notation has 4 similar symbols, being distinguished through different letters. The new notation adds the "Semantic Web Services" notation and a "Play" icon;

9. Send - It is not clear what the current notation is addressing. The new notation states clearly that the message is going to be sent elsewhere; 
T. Miranda et al.

10. Receive - It is not clear what the current notation is addressing. The new notation states clearly that the message is going to be received;

11. Action - Removed the round border. The clapperboard is enough to understand the semantic construct;

12. Message - The new notation attempts to be similar to the new notations adopted in "Message Sequence", "Send" and "Receive";

13. Message Sequence - Similar to the notations presented in "Send" and "Receive", the new notation hints a sequence of message being transmitted by those parties;

14. ODMOWLClass - The new notation is similar to the previous "Plan" symbol. It tries to remove two similar element from the visual notation (as the "Plan" symbol is totally different from the original one);

15. DomainRole - The current visual notation does not have any relation with a domain. The metaphor tried on the new notation aims at reflecting the web domains, inserting its roles on a web browser window;

16. Agent State - The current visual notation does not have any relation with an Agent State. The new notation attempts to add a "Secret Agent" to a typical rounded "State Icon" that appears on some loading screens;

17. Resource - The new notation reflects a box full of resources, which reflects more what the semantic construct is;

18. Web Service - The new notation adds a gear to an icon that relates to the web;

19. Grounding - Proposed by the MAS developers having experience on MAS and SWS;

20. Process - Proposed by the MAS developers having experience on MAS and SWS;

21. Interface - Proposed by the MAS developers having experience on MAS and SWS;

22. Precondition - Proposed by the MAS developers having experience on MAS and SWS;

23. Effect - The current visual notation does not have any direct relation with Effect. The new notation tries to adapt the "Magic" metaphor for an effect cause;

24. Architecture Role - The current visual notation does not have any direct relation with an "ArchitectureRole". The new icon adds the "Role" symbol to a common architecture plan;

25. Ontology Mediator Role - Proposed by the MAS developers having agent programming experience;

26. Semantic Web Organization (SWO) - The current visual notation does not have any direct relation with a SWO. The new symbol adds that relation;

27. Role Ontology - The new visual notation adapts to the new ODMOWLClass proposed above;

28. Organization Ontology - The new visual notation adapts to the new ODMOWLClass and "Semantic Web Services" proposed above;

29. Service Ontology - The new notation adapts to the new ODMOWLClass proposed above;

30. Interaction - Although it is perceptible what the current visual notation proposes, there is room for improvement by adding a clearer symbol;

31. Behavior - The current visual notation does not have any relation with the "Behavior" semantic construct. The new symbol tries to apply a metaphor related to the human behavior;

32. Agent Type - Proposed by the MAS developers having agent programming experience. 
Improving the Usability of a MAS DSML

\section{Evaluation}

\subsection{Experiment planning}

Goals Broadly, we aim to compare the impact of using the evolved version of the MAS DSML $\left(S E A_{-} M L++\right)$ when contrasted with the previous version ( $\left.S E A_{-} M L\right)$, focusing, one at a time, in different quality criteria for the language assessment. We present our evaluation goals following the GQM research goals template [2], which is shared among all our goals, with the exception of the term concrete quality criterion, which varies from one goal to the next.

In general, our goal is to analyse the effect of evolving from $S E A_{-} M L$ to $S E A_{-} M L++$, for the purpose of evaluation, with respect to the semantics transparency of the symbols used in the concrete syntax, from the viewpoint of researchers, in the context of an experiment conducted with participants with limited or no experience with MAS at Universidade Nova de Lisboa (UNL) in Portugal and EGE University in Turkey.

More specifically, our first goal is concerned about the comprehensibility of the symbols used on the concrete syntax, leading to the following formulation: Our first goal (G1) is to analyse the effect of evolving from $S E A_{-} M L$ to $S E A_{-} M L++$, for the purpose of evaluation, with respect to the comprehensibility of the symbols used in the concrete syntax, from the viewpoint of researchers, in the context of an experiment conducted with participants with limited or no experience with MAS at UNL and EGE University. Our second goal (G2) is concerned about the perceived usability of the concrete syntax. Our third goal (G3) is concerned about the effectiveness of the concrete syntax. Finally, our fourth goal (G4) is concerned about the efficiency.

All materials for the conducted evaluation, including experiment setup, result sets and statistics are also available in this chapter's online repository ${ }^{1}$

Tasks To achieve (G1), (1) each participant read and signed a consent letter regarding the data collected in the experiment. This letter was only used for the purpose of this study. All participants remained anonymous. Then (2) each participant selected the symbol (s)he found more suitable for each of the 33 $S E A_{-} M L++$ concepts identified in the PoN assessment reported in Section 4 . Finally, (3) participants filled in a background questionnaire.

We recruited a different, non-intersecting, group of participants for the remaining tasks. Again, (1) each participant read and signed a consent letter regarding the data collected, similar to the letter used in the other experiment. Then (2) each participant completed 4 exercises, 2 covering $S E A_{-} M L$ and 2 covering $S E A_{-} M L++$. Each exercise ended with the user filling in a questionnaire about it. We had a crossover design with 4 possible sequences, as represented in Table 3. The goal was to mitigate any potential learning effects and balance the number of participants working with each example in each of the possible sequence positions. Finally, (3) participants filled in a background questionnaire.

\footnotetext{
1 https://doi.org/10.5281/zenodo.1288390
} 
T. Miranda et al.

Table 3: Experimental design. Key: MT = Music Trading; EF = Expert Finder

\begin{tabular}{lllll}
\hline Sequence & Task 1 & Task 2 & Task 3 & Task 4 \\
\hline Group 1 & MT/SEA_ML++ & MT/SEA_ML++ EF/SEA_ML & EF/SEA_ML \\
Group 2 & EF/SEA_ML & EF/SEA_ML & MT/SEA_ML++ & MT/SEA_ML++ \\
Group 3 & MT/SEA_ML & MT/SEA_ML & EF/SEA_ML++ & EF/SEA_ML++ \\
Group 4 & EF/SEA_ML++ & EF/SEA_ML++ & MT/SEA_ML & MT/SEA_ML \\
\hline
\end{tabular}

Experimental material We provided each participant with a consent letter and a background questionnaire, which were the same for both experiments. In the symbol selection experiment, the participant also received a questionnaire where (s)he was asked to match each concept definition with the symbol that would best represent its concrete syntax. For the second experiment, the participants received 4 different scenarios with a corresponding challenge, each followed by a questionnaire about the notation they had just used. 2 of those scenarios were related with music trading among software agents, while the other 2 involved an agent-based expert finding system. Each of these scenarios had 2 versions, one with $S E A_{-} M L$ and the other with $S E A_{-} M L++$. Each participant received 2 different scenarios for each concrete syntax.

Participants Johnson 23] suggests that six individuals per subset of the population are the minimum required for a controlled experiment. It is sensible to take a larger number, but the costs should be kept to a minimum. Regarding the usability study, Nielsen 32 claims that testing with 5 people lets us find almost as many usability problems as by using many more test participants. However, when performing the quantitative studies, Nielsen suggests testing at least 20 users to get statistically significant numbers.

All the participants in our studies have formal University training in Informatics. For the symbol selection experiment, 25 participants (all undergraduate students) were involved. All of these participants are current or former students at Universidade Nova de Lisboa (UNL). 11 of those had some basic knowledge of MAS (in the context of a course), but not of SEA ML++. For the evaluation experiment, a total of 36 participants were included. That experiment was run in 2 replicas: The first one was conducted at UNL with 24 participants, including 12 with some basic knowledge of MAS. The second one was conducted at EGE University with 12 participants, all graduate students with some basic knowledge of MAS. All participants were selected through convenience sampling.

It is worth noting that the domain of agents interacting with SWSs is not an established professional occupation field and we could have limited number of researchers in the evaluation. Such professional evaluators are familiar with the concepts and their relations which makes the development and subsequently the evaluation more real. Because of this shortcoming, we have a small size society for the evaluation. 
Hypotheses, parameters and variables Overall, we hypothesize that the proposed $S E A_{-} M L++$ has a better concrete syntax than $S E A_{-} M L$. In order to make this more concrete, we anchor our formalized hypotheses on the research goals defined in Section 5.1. as presented in Table 4 For each of the high-level goals, we define the null $\left(H_{0} G i\right)$ and alternative $\left(H_{1} G i\right)$ hypotheses (where $i$ denotes the specific goal).

Table 4: Hypotheses

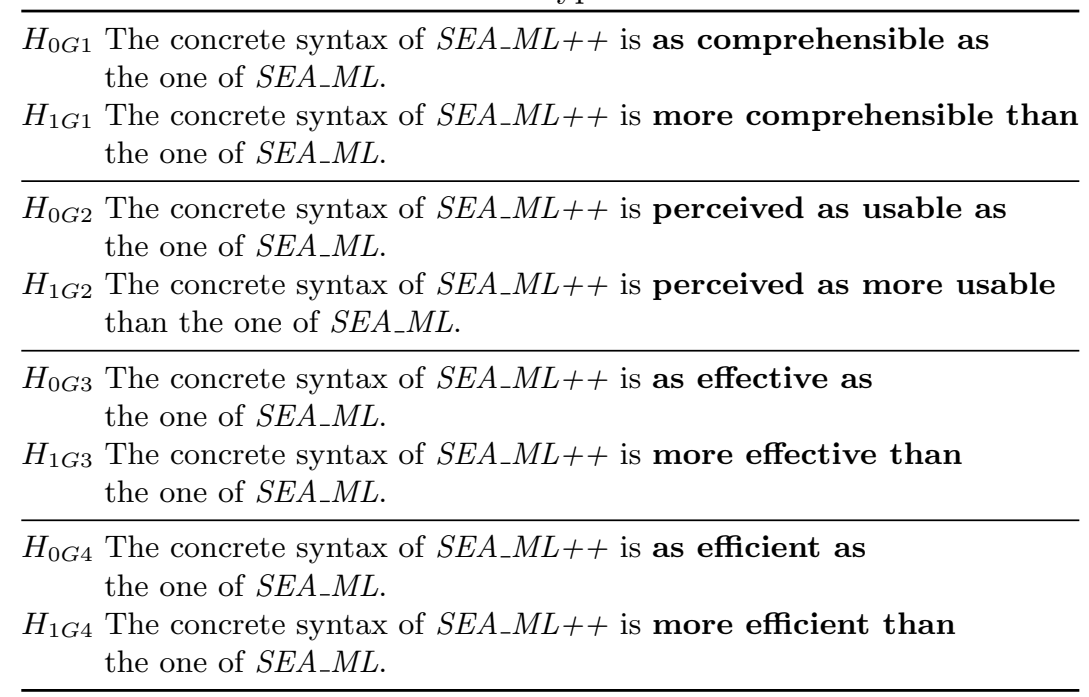

For all hypotheses, the independent variable is the concrete syntax, which can be $S E A_{-} M L++$ or $S E A_{-} M L$. The dependent variables are different for each of the tested hypothesis.

Comprehensibility. Graphical symbols' comprehensibility can be assessed by measuring hit rates, i.e., the percentage of correct responses 21.22. In this case, we measure the hit rate (percentage of answers where the correct symbol was chosen) for each concept in each of the concrete syntaxes.

Perceived Usability. In order to assess the perceived usability we asked our participants to fill in a System Usability Scale [5] questionnaire. This questionnaire consists of 10 questions, each with 5 response options, ranging from "Strongly Disagree" to "Strongly Agree". The scores are then converted to a scale of $0-100$. The threshold of 68 points is considered as the "average usability" [5]. Lower scores indicate below average usability, while higher scores are considered above average. In addition, we asked our participants to classify the following 3 statements:

- S1: The symbols on the user interface (UI) were easy to understand. 
T. Miranda et al.

- S2: The symbols on the UI are adequate to the MAS constructions they are linked to.

- S3: The symbols on the UI helped me solve the exercise in less time.

We deliberately used the term "symbols on the UI" (User Interface) rather than "concrete syntax", as a simplification for our participants, who were not necessarily familiar with the notion of "concrete syntax". For each of these sentences, the participants had to select from a five-point ordinal scale, ranging from 1 "Strongly Disagree" to 5 "Strongly Agree".

Effectiveness. We use the correctness of the answers of our participants to measure how effectively they were able to solve the exercises.

Efficiency. We recorded the duration of the working sessions to measure how fast our participants were able to complete their assigned tasks.

\subsection{Analysis}

Descriptive statistics In this section, we present descriptive statistics for the metrics collected to answer our research questions (Table 5). For each data row, we identify the corresponding goal (ranging from G1 to G4), the dependent variable (the quality focus for a particular goal), the independent variable, i.e. the concrete syntax followed by the descriptive statistics: the mean, standard deviation (SD), skewness (Skew), kurtosis (Kurt) and the p-value for the ShapiroWilk normality test $(S-W)$. In most of these variables, the assumption of normality is not reasonable $(p-$ value $<0.05)$, as confirmed by the visual inspection of boxplots in Figure 3, Q-Q plots and kernel density plots, omitted for the sake of brevity.

Table 5: Selection rate descriptive statistics

\begin{tabular}{|c|c|c|c|c|c|c|c|}
\hline \multicolumn{2}{|c|}{ Goal Dependent } & \multicolumn{6}{|c|}{ Independent Mdn. Mean S.Dev. Skew. Kurt. S-W } \\
\hline \multirow[t]{2}{*}{ G1 } & Preference & SEA_ML ++ & .44 & .45 & .14 & -.10 & .07 \\
\hline & & SEA_ML & .16 & .19 & .14 & .20 & -1.18 \\
\hline \multirow[t]{8}{*}{ G2 } & SUS & SEA_ML++ & 61.25 & 59.38 & 19.97 & -.20 & $\begin{array}{ll}-.24 & .409\end{array}$ \\
\hline & & SEA_ML & 57.50 & 54.17 & 20.62 & -.20 & .19 .268 \\
\hline & Understandability & SEA_ML++ & 4 & 3.96 & 1.09 & -1.15 & $.83 \quad .000$ \\
\hline & & SEA_ML & 3 & 2.92 & 1.25 & -.04 & $\begin{array}{ll}-.98 & .001\end{array}$ \\
\hline & Adequacy & SEA_ML++ & 4 & 3.65 & 1.02 & -.10 & $\begin{array}{ll}-1.10 & .000\end{array}$ \\
\hline & & SEA_ML & 3 & 2.96 & 1.03 & -.16 & $\begin{array}{ll}-.11 \quad .001 \\
\end{array}$ \\
\hline & Speed & SEA_ML++ & 4 & 3.83 & 1.10 & -.86 & $\begin{array}{ll}.23 & .000\end{array}$ \\
\hline & & SEA_ML & 3 & 2.85 & 1.29 & -.09 & $\begin{array}{ll}-.95 & .000\end{array}$ \\
\hline \multirow[t]{2}{*}{ G3 } & Correctness & 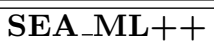 & 1.00 & .84 & . .32 & -1.763 & 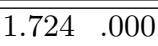 \\
\hline & & SEA_ML & 1.00 & .80 & .32 & -1.509 & $\begin{array}{ll}1.096 \quad .000\end{array}$ \\
\hline \multirow[t]{2}{*}{ G4 } & Duration & SEA_ML +十 & $11: 51$ & $13: 20$ & $06: 12$ & 1.520 & $2.434 \quad .000$ \\
\hline & & SEA_ML & $12: 24$ & $14: 48$ & 09:32 & 2.784 & $8.463 \quad .000$ \\
\hline
\end{tabular}


Improving the Usability of a MAS DSML
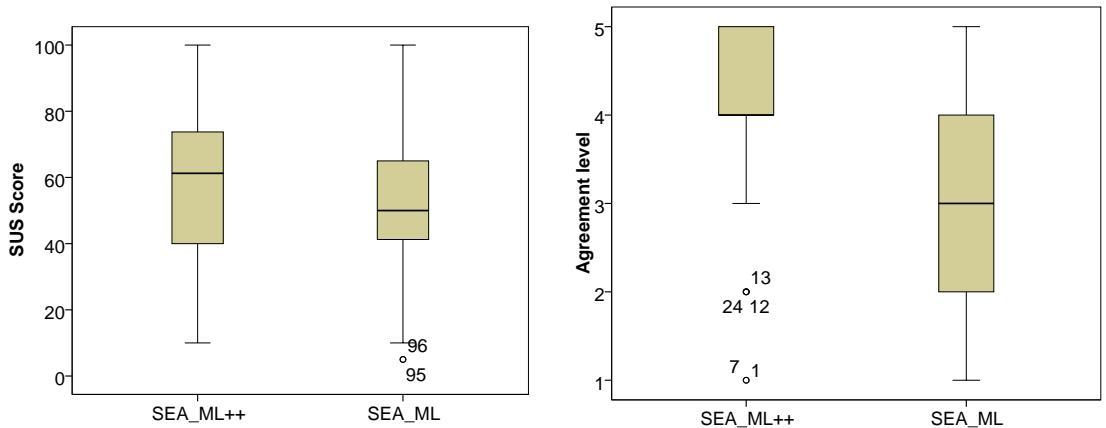

(a) SUS score

(b) Understandability
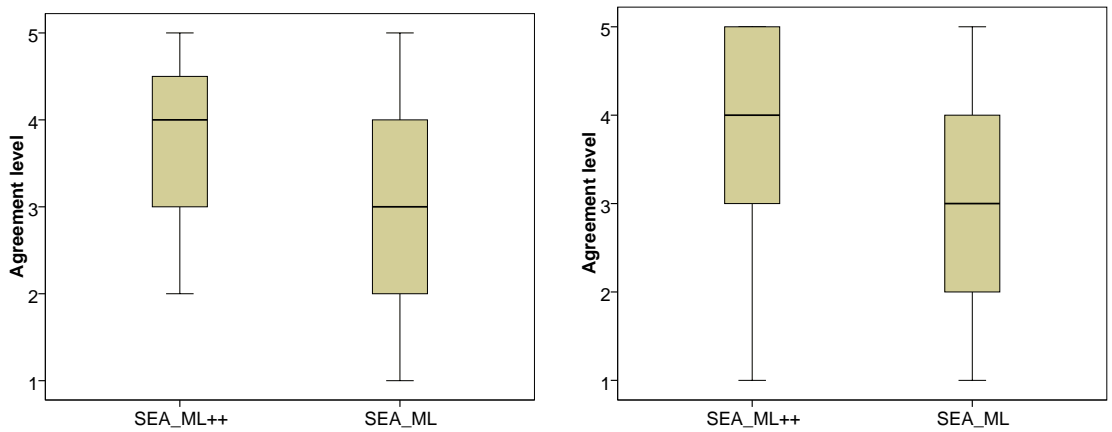

(c) Adequacy

(d) Speed

Fig. 3: Perceived Usability of SEA_ML vs SEA_ML++

Hypotheses testing We now present the results of our hypotheses tests.

G1: RQ1: Are participants more likely to select the correct elements from the PoN-based concrete syntax of $S E A_{-} M L++$ or the baseline $S E A_{-} M L$ concrete syntax elements? A Wilcoxon Signed-Ranks test was run and the output indicated that $S E A_{-} M L++$ scores $(M d n=.44)$ were statistically significantly higher than $S E A_{-} M L$ scores $(M d n=.16), Z=4.573, p<.001, r=.83$. This supports our hypothesis that participants were more likely to select the $S E A_{-} M L++$ elements.

G2: RQ2: Do participants using SEA_ML++ perceive it as more usable than $S E A \_M L$ ? In order to answer this question, we look at this from 2 different perspectives. We use a standard usability test - the System Usability Scale (SUS) - and a set of 3 questions to gather more detailed feedback (Figure 3 ).

SUS: Is $S E A_{-} M L++$ perceived as more usable than $S E A_{-} M L$ ? The usability did not differ significantly, according to Welch's t test, $t(141.854)=1.539, p=$ .126 from SEA_ML $++(M=59.38, S D=19.97)$ to the usability of SEA_ML $(M=54.17, S D=.20 .62)$ (Figure 3a). 
T. Miranda et al.

Understandability: The symbols on the user interface (UI) were easy to understand. Because the data was skewed for both variables, a Wilcoxon Signed-Ranks Test was run and the output indicated that $S E A_{-} M L++$ scores $(M d n=4)$, were statistically significantly higher than $S E A_{-} M L$ scores $(M d n=3), Z=3.683$, $p<.001, r=.53$ (Figure 3b).

Adequacy: The symbols on the UI are adequate to the constructs they are linked to. Because the data was skewed for both variables, a Wilcoxon SignedRanks Test was run and the output indicated that $S E A_{-} M L++$ scores $(M d n=$ 4) were statistically significantly higher than $S E A_{-} M L$ scores $(M d n=3), Z=$ 2.939, $p<.003, r=.42$. These results suggest that participants found $S E A_{-} M L++$ more adequate than $S E A_{-} M L$ to the constructs they were linked to (Figure 3c).

Speed: The symbols on the UI helped me solve the exercise in less time. Because the data was skewed for both variables, a Wilcoxon Signed-Ranks Test was run and the output indicated that $S E A_{-} M L++$ scores $(M d n=4)$ were statistically significantly higher than $S E A_{-} M L$ scores $(M d n=3), Z=3.324, p<.001$, $r=.48$ (Figure $3 \mathrm{~d}$ ). These results suggest that participants perceived using $S E A_{-} M L++$ had helped them solving the exercise faster than using $S E A_{-} M L$.

G3: We applied the Welch t-test, which is robust to deviations from normality within groups and when variance homogeneity among groups may not be assumed. The correctness does not differ significantly, according to Welch's ttest, $t(141.968)=.417, p=.519$ from the SEA_ML $(M=.80, S D=.32)$ to the SEA_ML $++(M=.84, S D=.32)$ concrete syntax. These results suggest that there was no difference between the 2 concrete syntaxes, in terms of complexity.

G4: As in G3, we applied the Welch t-test. The duration does not differ significantly, $t(122.030)=1.180, p=.280$ from the SEA_ML $(M=14: 48, S D=$ $09: 32)$ to SEA_ML++ $(M=13: 20, S D=06: 12)$ concrete syntax. These results suggest that there was no difference between the 2 concrete syntaxes, in terms of duration.

\subsection{Discussion}

Evaluation of the results and implications By using the PoN to guide a redesign of the concrete syntax of SEA_ML, we proposed SEA_ML++. We found that (RQ1) the participants in our study were better at correctly identifying the symbols with SEA_ML++. They found the SEA_ML++ syntax (RQ2) easier to understand, more adequate to the MAS constructs it represents and helpful for performing faster, when compared to the the SEA_ML syntax. However, in practice, (RQ3) participants were neither significantly able to use the language more correctly, (RQ4) nor significantly faster using it. So, overall, although the perception of language usage has improved with the new concrete syntax (and, with it, the developer experience), its implications for the actual usage of the language in agent development did not translate into improved effectiveness or efficiency (the small improvements observed were not significant). While it was certainly the case that there was room for improvement of the concrete syntax, the PoN-based improvements only took us as far as improving the perceived developer experience. Other alternative techniques, such as the sign production 
technique used successfully with other languages, such as $i^{*}[6]$, could potentially further improve the developer experience. That said, it seems more likely that the effectiveness and efficiency in using SEA_ML++ are mostly constrained by the semantics of the language. Further research is ongoing to explore this hypothesis.

Threats to validity The selection of participants is a potential threat. They are mostly representative of practitioners who are relatively inexperienced with MAS and, therefore, a good match for the main target population of this study. Most of the participants have less than 1 year experience on software agent development and only 5 participants in EGE University can be said experienced with having more than 3 years of MAS knowledge and implementation. As with many other languages, experts will cope better with the peculiarities of a given concrete syntax than newbies. The results obtained in the 2 replications were very similar, which increases our confidence on their external validity for other inexperienced MAS developers.

A second validity threat concerns the representativeness of the models used for this evaluation. While these models are good representatives of the complexity one would discuss with inexperienced MAS developers in the course of a training activity, further empirical evaluations with models of different complexities will increase the representativeness of this evaluation.

\section{Related Work}

In the last decade, several MAS modeling languages and DSMLs $4,9,11,14,17$ were proposed to support development of MASs. For example, DSML4MAS 19 introduces a general MAS metamodel with various viewpoints that enable the development of MAS for many application domains. As another example study, in 20], the authors develop a DSML and its supporting tool, called ERE_ML, for MAS working in emergency response environments. However, most of these DS(M)Ls proposed for MASs have been evaluated by just providing a case study demonstrating how the related language can be used for design and implementation of MAS. A quantitative analysis and/or qualitative evaluation considering e.g. the development time performance, generation performance, and/or the usability of the language are not considered in these studies.

In 8, an evaluation framework is proposed which provides the systematic assessment of both the language constructs and the use of agent DSMLs according to various dimensions and criteria. The study also provides an assessment of SEA_ML [7], however, it does not take into account the usability of the language, i.e. usefulness regarding the needs of language users. This evaluation framework is adopted in 26], 24] and 12] for the assessment of the proposed MAS DSMLs. Another MAS DSML evaluation feature exists in 3 for a textual DSL, JADEL, providing 4 abstractions, namely agents, behaviours, communication ontologies, and interaction protocols to JADE agent development framework. However, the study only evaluates JADEL's code generation performance. 


\section{T. Miranda et al}

The mentioned studies evaluate their MAS DS(M)Ls to some extent with or without using a structured evaluation framework. However, none of them addresses the usability of the MAS DS(M)Ls considering both the end-user perspective and the improvement of the visual language notation which, we argue that, is critical for the adoption of such languages in AOSE. In this sense, this study contribute to the literature by assessing the usability of an available MAS DSML, namely SEA_ML, and improving its new version.

In general, despite the fact that it is usually claimed that DSLs are more usable and leading to productivity gains, in 13 it has been identified a generalized lack of practice of reporting their usability assessment. The Software Language Engineering community has been seeking for adequate and systematic approaches to evaluating the usability of DSLs [1]. Work was reported [31] on how $i^{*}$ concrete syntax was evaluated using PoN and a new symbol set was proposed for it. In the sequence of this, in [6], it is compared the proposed concrete syntax with alternatives produced by novices (a stereotype and a prototype concrete syntaxes) and the standard $i^{*}$ concrete syntax.

Several modelling languages, for example, BPMN 2.0 [16], Use Case Maps [15], WebML [18, and misuse cases [34], use PoN to evaluate and identify improvement opportunities. It is possible to observe consistently similar conclusions concerning the challenges in most visual notations from a PoN perspective [30. Other studies assess the $i^{*}$ and KAOS modelling languages [27, using interviews, creation of models, and evaluation of those models and the modelling language and found clarity problems in the semantics definition of those languages.

\section{Conclusion and Future Work}

There are many modeling languages and DSMLs for MAS. Although there are a few studies addressing the evaluation of MAS DSMLs and their performances, the usability of these DSMLs is not investigated in a systematic way. In this study, the principles of The "Physics" of Notations are applied on a MAS DSML, called SEA_ML. By applying 9 principles, 43 notations of SEA_ML are evaluated and 32 of them are modified which are used in the development of the new version of SEA_ML called, SEA_ML++. In this way the notations in the graphical concrete syntax of the DSML are improved leading to the improvement of SEA_ML++. This hypothesis is examined under 4 research goals covering comprehensiveness, usability, effectiveness, and efficiency. The experiment conducted by the participants shows that the participants were more likely to select the SEA_ML elements and the symbols were easy to understand. However, the results show that there was no significant difference between the 2 concrete syntax, in terms of complexity and duration. Finally, it is worth indicating that this study mainly focuses on evaluating the use of notations/symbols in the DSML and does not cover the other issues (e.g. diagram complexity, scalability) which PoN can be utilized. These can be addressed in the future work. 
Improving the Usability of a MAS DSML

\section{Acknowledgment}

The authors would like to thank the followings: i) the Scientific and Technological Research Council of Turkey (TUBITAK) under grant 115E591, and ii) Portuguese grants NOVA LINCS Research Laboratory (Grant: FCT/MCTES PEst UID/ CEC/04516/2013) and DSML4MA Project (Grant: FCT/MCTES TUBITAK/0008/2014).

\section{References}

1. Barišić, A., Amaral, V., Goulão, M.: Usability Driven DSL development with USEME. Computer Languages, Systems and Structures (ComLan) 51, 118-157 (2017). https://doi.org/10.1016/j.cl.2017.06.005

2. Basili, V., Caldiera, G., Rombach, H.: Goal Question Metric Paradigm. Encyclopedia of Software Eng. 1, 528-532 (2001)

3. Bergenti, F., Iotti, E., Monica, S., Poggi, A.: Agent-oriented model-driven development for jade with the jadel programming language. Comput Lang Syst Str 50, 142-158 (2017)

4. Beydoun, G., Low, G., Henderson-Sellers, B., Mouratidis, H., Gomez-Sanz, J.J., Pavon, J., Gonzalez-Perez, C.: Faml: a generic metamodel for mas development. IEEE T Software Eng 35(6), 841-863 (2009)

5. Brooke, J.: Sus-a quick and dirty usability scale. Usability evaluation in industry 189(194), 4-7 (1996)

6. Caire, P., Genon, N., Heymans, P., Moody, D.L.: Visual notation design 2.0: Towards user comprehensible requirements engineering notations. In: RE'13. pp. 115124. IEEE (2013)

7. Challenger, M., Demirkol, S., Getir, S., Mernik, M., Kardas, G., Kosar, T.: On the use of a domain-specific modeling language in the development of multiagent systems. Eng Appl Artif Intel 28, 111-141 (2014)

8. Challenger, M., Kardas, G., Tekinerdogan, B.: A systematic approach to evaluating domain-specific modeling language environments for multi-agent systems. Software Qual J 24(3), 755-795 (Sep 2016)

9. Ciobanu, G., Juravle, C.: Flexible software architecture and language for mobile agents. Concurr Comp-Pract E 24(6), 559-571 (2012)

10. Da Silva, V.T., Choren, R., De Lucena, C.J.: Mas-ml: a multiagent system modelling language. IJAOSE 2(4), 382-421 (2008)

11. Demirkol, S., Challenger, M., Getir, S., Kosar, T., Kardas, G., Mernik, M.: Sea_l: a domain-specific language for semantic web enabled multi-agent systems. In: Computer Science and Information Systems (FedCSIS), 2012 Federated Conference on. pp. 1373-1380. IEEE (2012)

12. Faccin, J., Nunes, I.: A tool-supported development method for improved bdi plan selection. Eng Appl Artif Intel 62, 195-213 (2017)

13. Gabriel, P., Goulão, M., Amaral, V.: Do software languages engineers evaluate their languages? In: Proceedings of the XIII Congreso Iberoamericano en "Software Engineering" (CIbSE'2010) (2011)

14. Gascueña, J.M., Navarro, E., Fernández-Caballero, A.: Model-driven engineering techniques for the development of multi-agent systems. Eng Appl Artif Intel 25(1), 159-173 (2012)

15. Genon, N., Amyot, D., Heymans, P.: Analysing the cognitive effectiveness of the ucm visual notation. In: International Workshop on System Analysis and Modeling. pp. 221-240 (2010) 


\section{T. Miranda et al.}

16. Genon, N., Heymans, P., Amyot, D.: Analysing the cognitive effectiveness of the bpmn 2.0 visual notation. In: Proceedings of the Third International Conference on Software Language Engineering. pp. 377-396 (2010)

17. Gonçalves, E.J.T., Cortés, M.I., Campos, G.A.L., Lopes, Y.S., Freire, E.S., da Silva, V.T., de Oliveira, K.S.F., de Oliveira, M.A.: Mas-ml 2.0: Supporting the modelling of multi-agent systems with different agent architectures. J Syst Software 108, 77-109 (2015)

18. Granada, D., Vara, J.M., Brambilla, M., Bollati, V., Marcos, E.: Analysing the cognitive effectiveness of the webml visual notation. Softw Syst Model 16(1), 195227 (2017)

19. Hahn, C.: A domain specific modeling language for multiagent systems. In: Proceedings of the 7th international joint conference on Autonomous agents and multiagent systems-Volume 1. pp. 233-240 (2008)

20. Hosein Doost, S., Adamzadeh, T., Zamani, B., Fatemi, A.: A model-driven framework for developing multi-agent systems in emergency response environments. Softw Syst Model pp. 1-28 (2017)

21. ISO: Standard graphical symbols: Safety colours and safety signs-registered safety signs (iso 7010: 2003). International Standards Organisation (ISO): Geneva, Switzerland (2003)

22. ISO: Iso standard graphical symbols: Public information symbols (iso 7001:2007). International Standards Organisation (ISO): Geneva, Switzerland (2007)

23. Johnson, P.: Human computer interaction: psychology, task analysis, and software engineering. McGraw-Hill (1992)

24. Kardas, G., Bircan, E., Challenger, M.: Supporting the platform extensibility for the model-driven development of agent systems by the interoperability between domain-specific modeling languages of multi-agent systems. Comput Sci Inf Syst 14(3), 875-912 (2017)

25. Kardas, G., Gomez-Sanz, J.J.: Special issue on model-driven engineering of multiagent systems in theory and practice. Comput Lang Syst Str 50, 140-141 (2017)

26. Kardas, G., Tezel, B.T., Challenger, M.: Domain-specific modelling language for belief-desire-intention software agents. IET Softw 12(4), 356-364 (2018)

27. Matulevičius, R., Heymans, P.: Comparing goal modelling languages: An experiment. In: International Working Conference on Requirements Engineering: Foundation for Software Quality. pp. 18-32 (2007)

28. McGuinness, D.L., van Harmelen, F.: Owl web ontology language overview. w3c (2004)

29. Miranda, T.R.: Software Language Engineering : Interaction and Usability Modeling of Language Editors. MSc thesis, Universidade Nova de Lisboa, Faculdade de Ciências e Tecnologia, Monte Caparica, Portugal (2017)

30. Moody, D.: The "physics" of notations: toward a scientific basis for constructing visual notations in software engineering. IEEE T Soft Eng 35(6), 756-779 (2009)

31. Moody, D.L., Heymans, P., Matulevičius, R.: Visual syntax does matter: improving the cognitive effectiveness of the $i^{*}$ visual notation. Requir Eng 15(2), 141-175 (2010)

32. Nielsen, J.: How many test users in a usability study. Nielsen Norman 4(06) (2012)

33. Pokahr, A., Braubach, L., Walczak, A., Lamersdorf, W.: Jadex-engineering goaloriented agents. Developing multi-agent systems with JADE pp. 254-258 (2007)

34. Saleh, F., El-Attar, M.: A scientific evaluation of the misuse case diagrams visual syntax. Inform Software Tech 66, 73-96 (2015)

35. Shadbolt, N., Berners-Lee, T., Hall, W.: The semantic web revisited. IEEE Intell Syst 21(3), 96-101 (2006) 
Improving the Usability of a MAS DSML

\section{Appendix A. Descriptions of the Selected SEA_ML Concepts}

\begin{tabular}{|c|c|c|}
\hline Icon. & Concept & Description \\
\hline & $\begin{array}{l}\text { Semantic Web Agent } \\
\text { (SWA) }\end{array}$ & $\begin{array}{l}\text { Semantic web agent in the SEA_ML stands for each agent which is a } \\
\text { member of semantic web-enabled MAS. It is an autonomous entity } \\
\text { which can interact with both the other agents and the semantic web } \\
\text { services, within the environment. }\end{array}$ \\
\hline & $\begin{array}{l}\text { Semantic service } \\
\text { matchmaker agent } \\
\text { (SSMatchmakerAgent) }\end{array}$ & $\begin{array}{l}\text { It is a SWA extension. This meta-element represents matchmaker } \\
\text { agents which store the SWS' capabilities list in a MAS and compare it } \\
\text { with the service capabilities required by the other agents, in order to } \\
\text { match them. }\end{array}$ \\
\hline & Belief & $\begin{array}{l}\text { Beliefs represent the informational state of the agent, in other words } \\
\text { its knowledge about the world (including itself and other agents). }\end{array}$ \\
\hline & Goal & $\begin{array}{l}\text { A goal is a desire that has been adopted for active pursuit by the } \\
\text { agent. }\end{array}$ \\
\hline & Role & $\begin{array}{l}\text { An agent plays different roles to realize different behaviors in various } \\
\text { situations, such as organizations, or domains. }\end{array}$ \\
\hline & Capability & $\begin{array}{l}\text { Taking BDI agents into consideration, there is an entity called } \\
\text { Capability which includes each agent's Goals, Plans and Beliefs about } \\
\text { the surroundings. }\end{array}$ \\
\hline & Fact & $\begin{array}{l}\text { The statement about the agent's environment which can be true. } \\
\text { Agents can decide based on these facts. }\end{array}$ \\
\hline & Plan & $\begin{array}{l}\text { Plans are sequences of actions that an agent can perform to achieve } \\
\text { one or more of its intentions. }\end{array}$ \\
\hline $\mathbf{R}$ & $\begin{array}{l}\text { Semantic service register } \\
\text { plan } \\
\text { (SS_RegisterPlan) }\end{array}$ & $\begin{array}{l}\text { The Semantic Service Register Plan (SS_RegisterPlan) is the plan used } \\
\text { to register a new SWS by SSMatchmakerAgent. }\end{array}$ \\
\hline $\mathbf{F}$ & $\begin{array}{l}\text { Semantic service finder } \\
\text { plan } \\
\text { (SS_FinderPlan) }\end{array}$ & $\begin{array}{l}\text { Semantic Service Finder Plan (SS_FinderPlan) is a Plan in which } \\
\text { automatic discovery of the candidate semantic web services take place } \\
\text { with the help of the SSMatchmakerAgent. }\end{array}$ \\
\hline A & $\begin{array}{l}\text { Semantic service } \\
\text { agreement plan } \\
\text { (SS_AgreementPlan) }\end{array}$ & $\begin{array}{l}\text { Semantic Service Agreement Plan (SS_AgreementPlan) is a concept } \\
\text { that deals with negotiations on quality of service (QoS) metrics (e.g., } \\
\text { service execution cost, duration and position) and contract } \\
\text { negotiation. }\end{array}$ \\
\hline $\mathbf{F}$ & $\begin{array}{l}\text { Semantic service } \\
\text { executor plan } \\
\text { (SS_ExecutorPlan) }\end{array}$ & $\begin{array}{l}\text { After service discovery and negotiation, the agent applies the } \\
\text { Semantic Service Executor Plan (SS_ExecutorPlan) to invoke } \\
\text { appropriate semantic web services. }\end{array}$ \\
\hline & Send & $\begin{array}{l}\text { An action to transmit a message from an agent to another. This can be } \\
\text { based on some standard such as FIPA_Contract_Net }\end{array}$ \\
\hline & Receive & $\begin{array}{l}\text { An action to collect a message from an agent. This can be based on } \\
\text { some standard such as FIPA_Contract_Net }\end{array}$ \\
\hline $8=$ & Task & Tasks are groups of actions which are constructing a plan in an agent. \\
\hline & Action & An action is an atomic instruction which constitutes a task. \\
\hline & Message & $\begin{array}{l}\text { A package of information to be send from an agent to another; } \\
\text { possibly to deliver some information or instructions. Two special } \\
\text { types of actions, namely Send and Receive, are used to handle these } \\
\text { messages. }\end{array}$ \\
\hline
\end{tabular}




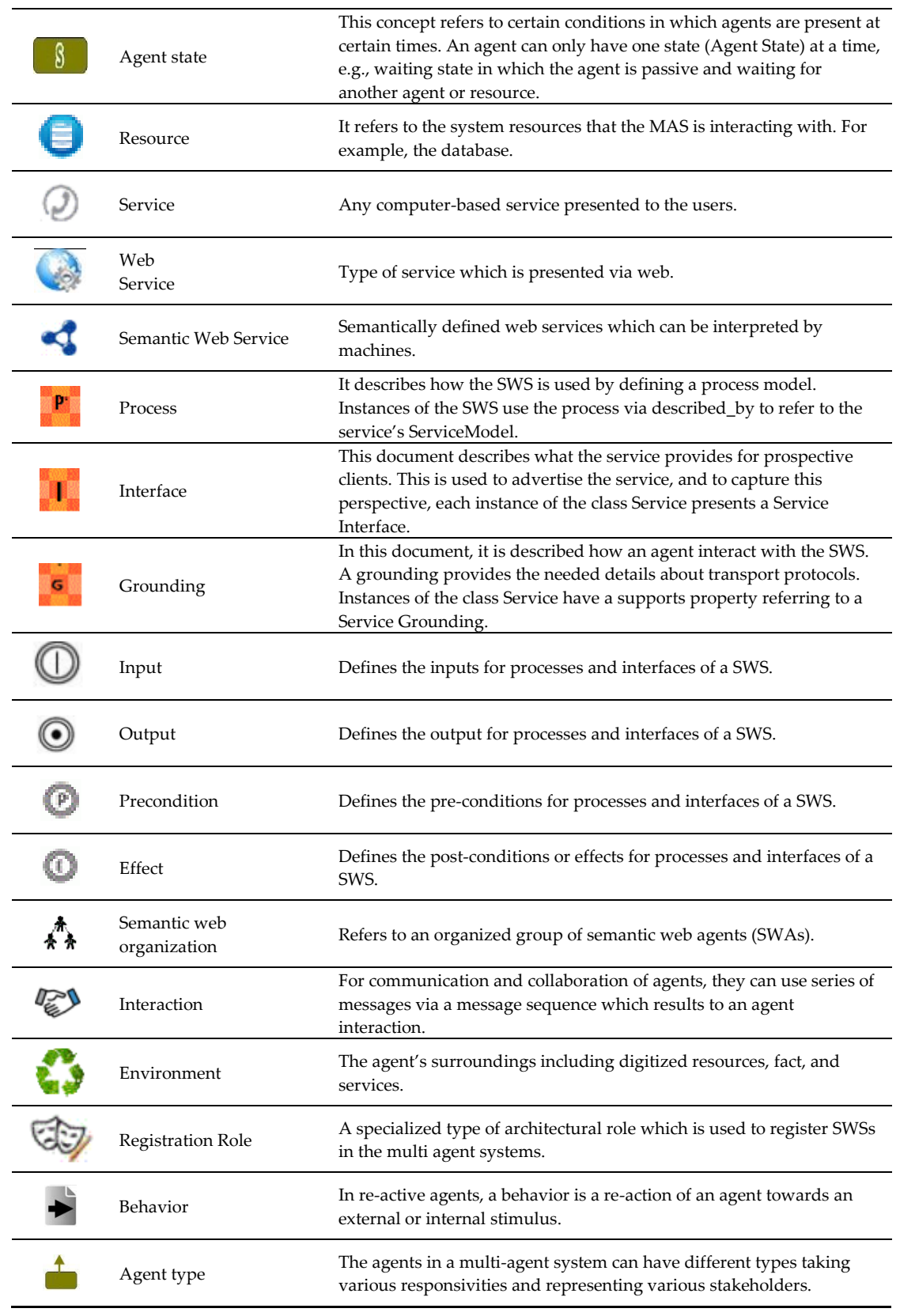

